\title{
Publisher's Editorial
}

For a full decade, Neuropsychobiology has participated in the quest to uncover and define the biological components of mental and behavioral disorders. Such a focus has brought the journal many of the same benefits and drawbacks faced by research in this field.

First, the prospect that psychiatric disorders might be managed through the chemical correction of a biological defect has proved enormously attractive to investigators in a wide range of disciplines. Apart from the sheer number of studies fueled by such a prospect, the field has attracted both especially talented investigators and the attention, expressed in funding, of the pharmaceutical industry. Perhaps most important, the approach of biological psychiatry has opened the mysteries of human mood and behavior to direct attack using some of the most powerful tools of modern biology.

At the same time, the very attractiveness of this line of inquiry has brought with it all the problems created by over-specialization and subspecialization. The situation might be compared to a large body of experts forever breaking up into smaller and smaller committees - and then getting so absorbed in details that they forget to reconvene. One frequently voiced complaint is that researchers in this field seem to forget, or dismiss as irrelevant, all that has been done in psychiatry and psychology. For their part, many psychologists and psychiatrists tend to view the biological approach as reductionistic - as though the 'heart and soul' of human behavior could be reduced to a chain of neurochemical events, denying the importance of life events and the social environment. 
For these reasons, Neuropsychobiology introduced a new editorial concept in 1983. For the past two years, the journal has covered developments in four distinct, but closely interrelated areas. Whereas the section on 'Biological Psychiatry' continues its vital and diversified attack on psychiatric illness, readers are reminded, in the section on 'Biological Psychology/Pharmacopsychology', of the importance of understanding the psychology of normal mental functions. The section on 'Pharmacopsychia-try' keeps the reader in touch with drug research and potential practical applications, while the section on 'Pharmacoelectroencephalography' provides an update of what is happening with a new technology applicable to all other areas of research on either normal or disturbed functions.

The editors and publisher hope that such an approach will go some way towards integrating work in the neuro-sciences while, at the same time, letting each individual section pursue the directions of its own natural course.

We thank the many contributors who have supported Neuropsychobiology in its efforts to perform an integra-tive function. As the journal continues to grow, future issues will also be including state-of-the-art reviews, short communications on experimental design or methodology, and editorials in areas where work is either controversial or confusing. Contributions are always welcome and should be sent to the attention of the editor in charge of the most appropriate section.

Thomas Karger, Basel 